\title{
Pathologies En-Route of Oral Basaloid Malignancies to Basaloid Squamous Cell Carcinoma
}

Mehendiratta $\mathrm{M},{ }^{1}$ Rehani $\mathrm{S},{ }^{1}$ Kumra $\mathrm{M},{ }^{1}$ Shah $\mathrm{MK}^{2}$

\author{
${ }^{1}$ Department of Oral and Maxillofacial Pathology \\ Sudha Rustagi Dental College and Research Institute \\ Haryana \\ ${ }^{2}$ Department of Hematology, St. Stephen's Hospital \\ Delhi, India
}

\author{
Corresponding Author \\ Monica Mehendiratta \\ Department of Oral and Maxillofacial Pathology \\ Sudha Rustagi Dental College and Research Institute \\ Haryana, India \\ E-mail: monicamehendiratta@gmail.com
}

\section{Citation}

Mehendiratta M, Rehani S, Kumra M, Shah MK. Pathologies En-Route of Oral Basaloid Malignancies to Basaloid Squamous Cell Carcinoma. Kathmandu Univ Med J 2014;46(2):141-5.

\begin{abstract}
Accurate histopathological diagnosis of any tumour is imperative because of variable prognostic and clinical implications. Basaloid squamous cell carcinoma (BSCC) is a rare distinctive histological variant of oral squamous cell carcinoma (OSCC) exhibiting an aggressive clinical course and poor prognosis. The advanced stage of presentation is speculatively responsible for this biological behaviour which is manifested by development of metastasis and subsequently poor survival. However, the non-specific clinical appearance and histopathological resemblance of BSCC with OSCC and other oral basaloid malignancies (OBM) especially in limited sample size poses diagnostic difficulties. The article emphasizes on the diagnostic criteria and pitfalls encountered in making the differential diagnosis of BSCC from OBMs along with report of a case presented at a dental college in January 2012.
\end{abstract}

\section{KEY WORDS}

Basaloid squamous cell carcinoma, differential diagnosis, oral basaloid malignancy, oral squamous cell carcinoma

\section{INTRODUCTION}

Appropriate investigations, treatment and management of oral squamous cell carcinoma (OSCC) demand a critical protocol to follow which involves a multi-disciplinary approach of various specialities of health sciences. The treatment protocols for it are becoming either dependent on the clinical staging of a tumour which might bring about unwanted mutilation and morbidity or under-estimation and mortality. One of the important determinants for the prediction of the prognosis and appropriate therapeutic regime of OSCC is its categorization into its clinicopathological variant. These variants include verrucous carcinoma, spindle cell carcinoma, basaloid squamous cell carcinoma (BSCC), nasopharyngeal carcinoma; adenosquamous cell carcinoma, adenoid squamous cell carcinoma, undifferentiated carcinomas etc. They display different growth patterns, histopathological features and biological behaviour from conventional OSCC. ${ }^{1}$

Amongst these, BSCC is considered an aggressive tumour whereas verrucous carcinoma displays indolent behaviour with lowest invasive and metastatic potential. ${ }^{1}$ BSCC was first described by Wain et al in 1986 arising in the upper aero-digestive tract. $^{2}$ Although topographically, BSCC of head and neck has a peculiar site distribution but due to its non-distinct clinical and macroscopic aspect, similar etiological risk factors including its questionable association with HPV as that of OSCC, the diagnosis definitely becomes difficult for the clinicians and adds miseries to pathologists roles. $^{3-5}$ The correct diagnosis is important as it was considered to be high grade in the earlier reports. However, some debatable matter exists concerning the prognosis as recent data indicates a similar course if diagnosed at an early stage..$^{2,3,6-8}$ Moreover, most BSCCs are diagnosed at advanced clinical stages leading to poor overall patient survival rates and thus, an unfavourable global prognosis. ${ }^{7}$

Microscopically, BSCC is characterized by an invasive neoplasm composed of nests and/or lobules of basaloid cells with peripheral nuclear palisading, intimately associated with a dysplastic squamous epithelium/carcinoma in-situ 
and/or invasive OSCC. ${ }^{2}$ The overlapping histopathological resemblance of BSCC with OBM e.g., Adenoid cystic carcinoma (ACC); small cell neuroendocrine carcinoma (SCNC); HPV-related Non-keratinized carcinoma (NKCa) and basal cell adenocarcinoma (BCA) may lead to erroneous diagnosis especially in an incisional/representative biopsy sample.

This article is written with an intention, firstly to alarm clinicians about this clinically indistinct variant of OSCC which is "so-called" aggressive. Secondly, to aware histopathologists about other OBMs as the treatment protocol or prognosis amongst these entities is disparate. Thus, BSCC should be cautiously distinguished from other OBMs.

\section{CASE REPORT}

Forty two years old, woman presented with a painful ulcer on the lateral border of the tongue from two years duration at the department of Oral Pathology and Microbiology at Sudha Rustagi Dental College, Haryana, India in January 2012. Patient reported sudden rapidity of growth and pain from past few months. On clinical examination, a firm, ulcero-proliferative growth of 2.0 to $3.0 \mathrm{~cm} 2$ was seen (Fig 1). Regional lymph-nodes were non-palpable. Patient revealed no history of smoking or any habit and her past

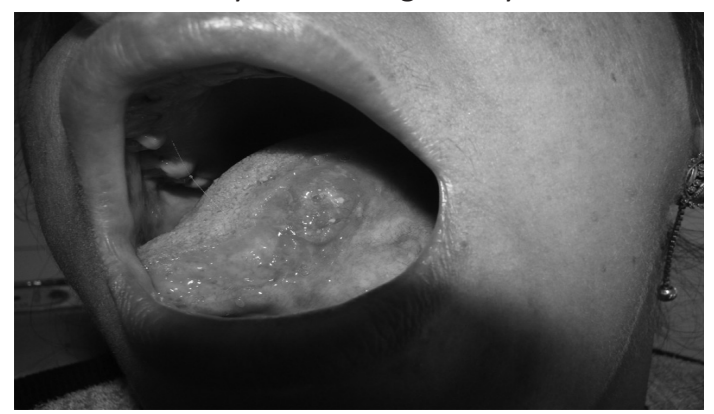

Figure 1. Ulcero-proliferative growth on the lateral border of tongue.

dental and medical history were unremarkable. Blood examinations were found insignificant. At this stage, a provisional diagnosis of OSCC was made and was graded as T1N0M0. After obtaining a written consent from the patient, an incisional biopsy under local anaesthesia was performed.

The tissue was routinely fixed, processed and stained with hematoxylin and eosin. The histopathological examination revealed closely apposed lobules and thin trabeculae of dysplastic basaloid cells with few squamoid cells. The epithelial tumor cells exhibited abundant nuclear and cellular pleomorphism, mitotic figures and peripheral nuclear palisading (Fig 2). Epithelial tumor islands exhibiting large central foci of comedo-necrosis (Fig 3) and few tumor cells infiltrating degenerating muscle tissue can be seen in connective tissue. The overlying epithelium showed areas of carcinoma in-situ in abrupt conjunction with an apparently normal hyperplastic stratified squamous epithelium

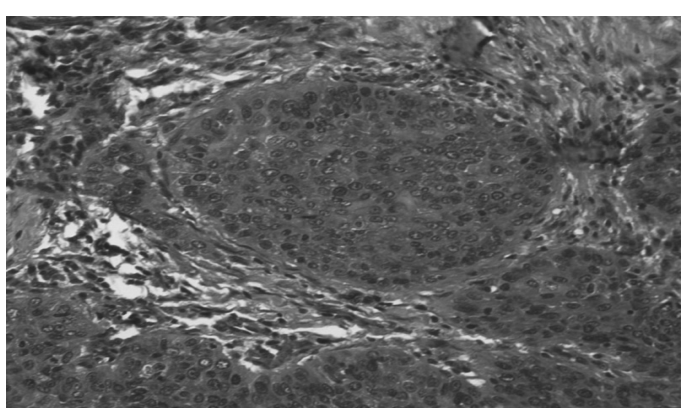

Figure 2. Basaloid tumor islands exhibiting nuclear and cellular pleomorphism and peripheral nuclear palisading.

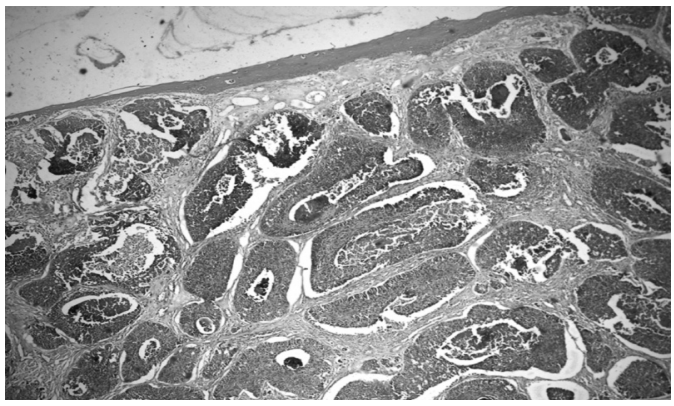

Figure 3. Invading basaloid nests and strands with comedonecrosis can be seen.

(Fig 4). The intervening stroma was highly cellular with mixed inflammatory cell infiltrate. Immunohistochemistry showed cytoplasmic and membranous positivity for CAM 5.2 (Fig 5).

BSCC was reported in the incisional biopsy report and this was followed by further examination and wider excision. The excisional biopsy confirmed the histopathological diagnosis.

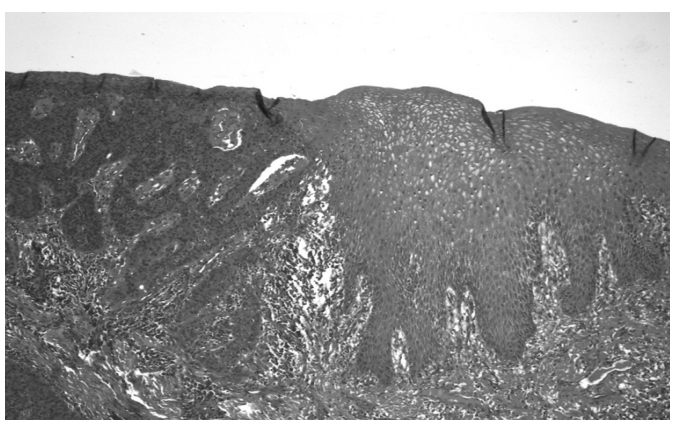

Figure 4. Abrupt junction between carcinoma-in-situ and hyperplastic stratified squamous epithelium.

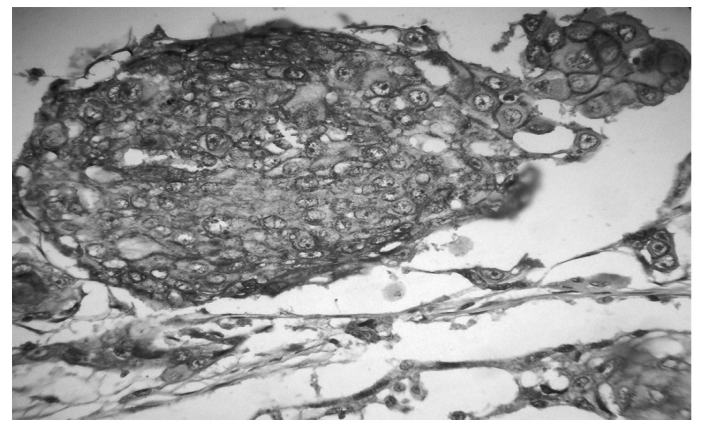

Figure 5. Membrane and cytoplasmic positivity for CAM 5.2. 


\section{DISCUSSION}

BSCC is a rare epithelial malignant tumor which shows a strong preference for upper aero-digestive tract and thus, presents location-specific clinical signs and symptoms. In the oral cavity, often seen on the base of tongue and tonsils, other sites involved are floor of mouth, gingiva and buccal mucosa. ${ }^{6}$ Oral BSCC reportedly occurred almost equally in both sexes often between $60-80$ years of age. , $^{4,10}$ The phenomenon of older age is more obvious in BSCC than in conventional SCC because of its late diagnosis. The carcinoma grows rapidly and is associated with pain which is responsible for its shorter history. ${ }^{11}$

Similar to SCC, tobacco and/or alcohol consumption have been found in $80 \%$ of cases and HPV's association are proven to be strong risk factors. ${ }^{4,5,9}$ The unique presentation of our case was its location, that is, lateral border of the tongue which has not been reported as per our knowledge.

BSCC shows a biphasic phenotype, i.e. basaloid and squamous, which often displays an abrupt transition between them. Smooth contoured, solid-lobules of closely packed small cells with hyperchromatic nuclei, and scant cytoplasm are characteristic findings. Comedotype necrosis, mitotic figures and prominent peripheral palisading are frequent features. Basaloid cells arranged in small cystic spaces or pseudoglandular pattern and stromal hyalinization can be seen unlike conventional OSCC. The squamoid component of BSCC can be either in-situ carcinoma, or invasive keratinizing OSCC. The latter is usually located superficially; it may also present as a focal squamous differentiation within the basaloid tumour islands. ${ }^{2,6,9,10}$

The squamous differentiation is identified by the presence of two or more of the following histological features: (i) individual cell keratinization, (ii) intercellular bridges, (iii) keratin pearl formation, and (iv) cells arranged in a pavementing pattern. ${ }^{12}$ The intercellular deposition of PAS- and Alcian blue positive material, eosinophilic hyaline or mucohyaline material simulates the appearance of reduplicated basement membrane. ${ }^{13}$ In the earliest review by Wain, et al. (1986) features of basaloid component is categorized into four principal histologic features. ${ }^{2}$

Immunohistochemically, the epithelial origin is confirmed by diffuse staining in squamoid and basaloid component by cytokeratin AE1/AE3, EMA and CAM 5.2 in BSCC. Unlike OSCC, CK-13 positive staining was limited to the area of well differentiated squamous cells with basaloid cells showing no immunoreactivity. The active cell proliferation, as demonstrated by higher PCNA index, high labelling scores for p53 and Ki67, histologically high mitotic rate, and comedo-like necrosis suggest the high grade malignant nature of BSCC. Low expression of p27 and E-cadherin correlated independently with poor prognosis. ${ }^{11,12}$

\section{Differential Diagnosis}

Histopathological diagnosis of BSCC could be easily made when the architecture can be adequately and clearly assessed in the resected tumor bulk with aid of its potentially helpful clinical features. However, in the incisional/representative biopsies the evaluation can be quite challenging. The differential diagnosis of BSCC embraces all the oral basaloid malignancies (OBM) which are characterized by round to oval nuclei with a scanty cytoplasm (Fig 6).

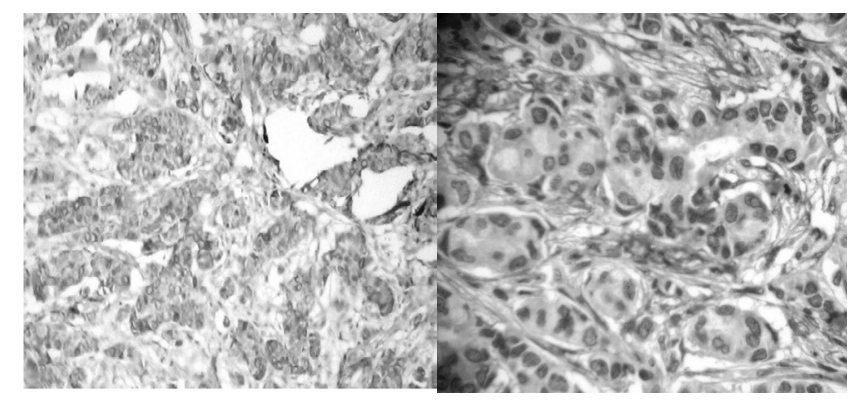

Figure 6. Distinguishing features of oral basaloid malignancies.

1. The trickiest differential diagnosis is the solid variant of Adenoid cystic carcinoma (ACC) for which extensive work needs to be done both cytologically and histologically. As emphasized by Ide F, et al (2002), several of the oral tumours reported as "solid ACC" were bona fide examples of BSCC. ${ }^{10}$ ACC is usually well-treated with only excision of the primary tumor but the solid pattern has fulminant course with earlier metastases and poorer survival statisticswhereas, $\mathrm{BSCC}$ requires radical management regime thus making its differential diagnosis from ACC critical. ${ }^{12,14,15}$

Common features such as the basaloid cytological morphology, cystic, glandular-like spaces and intercellular amorphous, eosinophilic, hyaline deposits are seen in both the malignancy. ${ }^{12}$ To differentiate, clinically the BSCC are often associated with cervical lymph node metastasis which is rare in ACC. . $^{412}$

Histologically, the PAS positive microcystic spaces in basaloid lobules of BSCC akin to solid ACC are generally limited though high-grade nuclear pleomorphism, mitoses and comedo-necrosis is high. ${ }^{4,10}$ Solid ACC nearly always includes well-formed tubular structures and show evidences of minor salivary glands which is less likely to be seen in BSCC. ${ }^{4,10,12,16}$ Essential diagnostic feature is the presence of squamous differentiation or the foci of SCC or carcinomatous change in the surface epithelium of BSCC. ${ }^{10}$ Immunohistochemically, the presence of myoepithelial cells in ACC and its positivity for S-100 and SMA helps differentiating from BSCC. ${ }^{11}$

2. The second important differential diagnosis would be Small cell neuroendocrine carcinoma (SCNC) which constitutes a very rare group affecting the non-keratinized oral mucosa. It exhibits an extremely adverse prognosis with tendency to develop distant metastases and very short survival. ${ }^{12,17} \mathrm{SCNC}$ is a diagnostic challenge and have been apparently misdiagnosed as poorly differentiated adenosquamous cell carcinoma or squamous cell carcinoma 
in the past due to the lack of molecular pathological analysis. ${ }^{17}$ This is an important differential diagnosis as it is often non-surgically treated with radiation and systemic chemotherapy. ${ }^{18}$

Histopathologically, both these show sheets of basaloid appearing cells and rosette-like structures. ${ }^{15}$ Frequently BSCC exhibits lobular pattern, hyalinosis, stromal mucin, cyst-like spaces and peripheral palisading which are absent in SCNC. ${ }^{12}$ Unlike BSCC, SCNC shows characteristic nuclear moulding and often crushing artefacts. No squamous element which is particularly important to establish the diagnosis of BSCC are seen in SCNC. ${ }^{15}$ Immunohistochemically, SCNC is positive for all the neuroendocrine markers such as synaptophysin and chromogranin and ultrastructurally, shows evidence of neurosecretory granules. ${ }^{4,11,12}$ Both SCNC and BSCC react to pancytokeratin antibodies AE1/AE3 and CAM 5.2 but in SCNC it is frequently seen in a paranuclear pattern. ${ }^{12}$

3. HPV-related nonkeratinizing carcinoma (NKCa) is a distinct molecular, pathological and clinical disease entity from non-HPV driven keratinizing SCC. ${ }^{19}$ When compared to BSCC or conventional keratinizing carcinoma, it is biologically less aggressive, typically responds more favourably to organ-sparing treatment modalities with better prognosis. Thus, distinction between these two variants is important. ${ }^{12,19}$ Clinically, when compared with BSCC, NKCa occurs at a relatively younger age. PCR, ISH, etc have consistently demonstrated the presence of HPV genome in NKCa whereas the association of HPV with BSCC is controversial. ${ }^{5}$

The basaloid morphology, brisk mitotsis, comedonecrosis, sometimes peripheral palisading in the cords, nests and sheets of basaloid cells of HPV-related nonkeratinizing carcinoma when occurs at the base of tongue makes it an important differential for BSCC. ${ }^{12,19}$ Similar to BSCC, the diagnosis can be delayed due to non-detection of small primary tumor and metastasis to cervical lymph nodes. For these reasons, histologically, cases of NKCa are occasionally misdiagnosed as BSCC. ${ }^{12}$

Microscopically, NKCa shows basaloid morphology, but unlike BSCC, it never shows cystic spaces with mucinlike material, coagulative necrosis and stromal hyalinosis with basement membrane-like material. Also, NKCa is

\section{REFERENCES}

1. Pereira MC, Oliveira DT, Landman G, Kowalski LP. Histologic subtypes of oral squamous cell carcinoma: prognostic relevance. J Can Dent Assoc 2007 May; 73(4):339-44.

2. Wain SL, Kier R, Vollmer RT, Bossen EH. Basaloid-squamous carcinoma of the tongue, hypopharynx, and larynx: report of 10 cases. Hum Pathol 1986; 17(11):1158-166.

3. Winzenburg SM, Niehans GA, George E, Daly K, Adams GL. Basaloid squamous carcinoma: a clinical comparison of two histological types with poorly differentiated squamous cell carcinoma. Head Neck Surg 1998; 119(5):471-475. not associated with conventional SCC or areas of frank keratinisation. ${ }^{12,19}$ Immunohistochemically, NKCa stains strongly with p16, negative or weakly with p53 whereas BSCC shows strong staining with $p 53 .^{20}$

4. Basal cell adenocarcinoma (BCA) is a low-grade major salivary gland malignancy with relatively favourable behaviour but has been reported intra-orally even in the upper-aeordigestive tract akin to BSCC. ${ }^{21}$

Presence of basaloid cells, and their arrangement in a solid or trabecular pattern may emulate those of BSCC. ${ }^{22}$ In BCA however; the basaloid cell population has two cell types, large pale and small dark cells. ${ }^{10}$ Features like focal necrosis, mitotic activity, squamous differentiation and peripheral palisading are less frequent and less prominent in BCA and BSCC shows squamous component. ${ }^{10,11} 14,21$ Immunohistochemically, these small dark cells are variably positive for myoepithelial markers in different cases e.g., keratin, vimentin, muscle specific actin, p63, and myosin. ${ }^{17}$

\section{CONCLUSION}

Histopathological distinction of a tumor is significant especially when tumors are aggressive as the window of opportunity to act early is very narrow or absent. Ironically, BSCC is still labelled as an aggressive tumor despite the various recent studies proving its similar clinical course as conventional OSCC when site and stage are matched. Although the oral cavity is accessible for dental examination to detect early signs of oral cancer, significant delays seem to occur in the diagnosis of BSCC as it has a predilection in the hidden areas i.e., the oro-pharyngeal region. Thus, the clinicians should aim do thorough oral examinations for the detection of these tumors and to provide the best chance for survival.

Also, the pathologists should keep the following features in mind while diagnosing OBM:

1) Cytologically and histologically, dual composition i.e., basaloid and squamoid elements,

2) Overlying epithelium displaying carcinoma in-situ, connected with invading tumor and keratinizing squamous cell carcinoma component,

3) Abundant nuclear pleomorphism.

4. Ereño C, Gaafar A, Garmendia M, Etxezarraga C, Bilbao FJ, López JI. Basaloid Squamous Cell Carcinoma of the Head and Neck. A Clinicopathological and Follow-Up Study of 40 Cases and Review of the Literature. Head and Neck Pathol 2008; 2(2):83-91.

5. Chernock RD, Lewis JS Jr, Zhang Q. El-Mofty Sk. Human papillomavirus positive basaloid squamous cell carcinomas of the upper aerodigestive tract. Hum Pathol 2010 Jul; 41(7):1016-23. Epub 2010 Mar 17.

6. Ferlito A, Altavilla G, Rinaldo A, Doglinio C. Basaloid squamous cell carcinoma of the larynx and hypopharynx. Ann Otol Rhinol Laryngol 1997; 106(12):1024-35. 
7. Morales-Puebla J M, Toro-Rojas M, Segura-Saint-Gerons R, FanegoFernández J, López-Villarejo P. Basaloid squamous cell carcinoma: Report of five cases. Med Oral Patol Oral Cir Bucal 2010 May 1; 15 (3):e451-5.

8. De Sampaio Góes FC, Oliveira DT, Dorta RG, Nishimoto IN, Landman G, Kowalski LP. Prognoses of oral basaloid squamous cell carcinoma and squamous cell carcinoma. A comparison. Arch Otolaryngol Head Neck Surg 2004; 130 (1):83-86.

9. Cardesa A, Zidar N, Ereno C. Basaloid squamous cell carcinoma. In: World Health Organization Classification of Tumors. Pathology and Genetics of Head and Neck Tumors. Barnes L, Eveson JW, Reichart P, Sidransky D (eds), International Agency for Research on Cancer (IARC) Press. Lyon: France; 2005.pg 124-5.

10. Ide F, Shimoyama T, Horie N, Kusama K. Basaloid squamous cell carcinoma of the oral mucosa: a new case and review of 45 cases in the literature. Oral Oncol 2002; 38(1):120-124.

11. Yu GY, Gao Y, Pang X. A clinicopathologic study on basaloid squamous cell carcinoma in the oral and maxillofacial region. Int. J. Oral Maxillofac. Surg 2008; 37(11): 1003-8 Epub 2008 Jul 15.

12. Barnes L, ed. Surgical Pathology of the Head and Neck. 3rd ed. New York; Informa Healthcare: 2009

13. Weidner N, Cote RJ, Suster S, Weiss LM. Modern Surgical Pathology. 2nd ed. Philadelphia, PA,; WB Saunders Co: 2003

14. Ellis GL, Auclair PL, Gnepp DR, eds. Surgical pathology of the salivary glands. Philadelphia, Pa; Saunders: 1991.
15. Vasudev $P$, Boutross-Tadross $O$, Radhi J. Basaloid squamous cell carcinoma: two case reports. Cases J 2009 Dec 18; 2:9351.

16. Jain K, Harshaminder K, Madhushankari GS. Basaloid Squamous Cell Carcinoma in Retromolar Ridge Area: A Rare Case Report. Int J Oral Maxillofac Pathol 2011; 2(3):27-31.

17. Mahomed F. Neuroendocrine cells and associated malignancies of the oral mucosa: a review. J Oral Pathol Med 2010 Feb; 39(2):121-7. Epub 2009 Dec 8.

18. Wenig BM. Squamous Cell Carcinoma of the Upper Aerodigestive Tract: precursors and problematic Variants. Mod Pathol 2002;15(3):229-254

19. Chernock RD, El-Mofty SK, Thorstad WL, Parvin CA, Lewis JS J. HPVrelated nonkeratinizing squamous cell carcinoma of the oropharynx: utility of microscopic features in predicting patient outcome. Head Neck Pathol 2009 Sep; 3(3):186-94.

20. Rodriguez Tojo MJ, Garcia Cano FJ, Infante Sanchez JC, veazquez Fernandez E, Aquirr Urizar JM. Immunoexpression of p53, Ki-67 and E-cadherin in basaloid squamous cell carcinoma of the larynx. Clin transl Oncol 2005 Apr; 7(3):110-4.

21. Parashar P, Baron E, Papadimitriou JC, Ord RA, Nikitakis NG. Basal cell adenocarcinoma of the oral minor salivary glands: review of the literature and presentations of two cases. Oral Surg Oral Med Oral Pathol Oral Radiol Endod 2007 Jan; 103(1):77-84.

22. Shinno $Y$, Nagatsuka $H$, Siar $C H$, Tsujigiwa $H$, Tamamura R, Gunduz $M$, et al. Basaloid squamous cell carcinoma of the tongue in a Japanese male patient: A case report. Oral Oncol extra 2005; 41:65-69. 